\title{
Genetic Heterogeneity in Patients with X-Linked Recessive Chronic Granulomatous Disease
}

\author{
TADASHI ARIGA, MASANORI NAKANISHI, KAZUHIRO TOMIZAWA, SHINOBU IMAJOH-OHMI, \\ SHIRO KANEGASAKI, YUKIO SAKIYAMA, AND SHUZO MATSUMOTO \\ Department of Pediatrics, Hokkaido University School of Medicine, Sapporo, Japan /T.A., M.N., K.T., Y.S., \\ S.M.J; and Institute of Medical Science, the University of Tokyo, Japan [S.I-O., S.K.]
}

\begin{abstract}
Genetic heterogeneity in 12 patients from 11 different families with $\mathrm{X}$-linked recessive chronic granulomatous disease was studied by Southern blot analysis using cytochrome $b$ heavy-chain cDNA as a probe. We found the abnormal restriction length fragment patterns of the cytochrome $b$ heavy-chain gene in three families, which were not observed in healthy controls. DNA from one patient showed the abnormal patterns after digestion with several restriction enzymes. The DNA of two other patients showed the abnormality only with TaqI and PstI. Analysis of the same family members indicated that these abnormal patterns cosegregated with the disease. The other nine patients from eight families did not have any abnormalities detectable by Southern blot analysis. Although further experimentation should be done to study the molecular genetic heterogeneity in most $\mathrm{X}$-linked chronic granulomatous disease families (eight of 11), we were able to demonstrate at least three different types of mutations in the cytochrome $b$ heavy-chain gene responsible for the disease. (Pediatr Res 31: 516-519, 1992)
\end{abstract}

\section{Abbreviations}

CGD, chronic granulomatous disease

CGD is an uncommon inherited disorder characterized clinically by recurrent pyogenic infections (1). In the majority of the families (65 to $70 \%$ ), CGD is inherited in an X-linked pattern; in the remainder of families the mode of inheritance is autosomal recessive (2). The high susceptibility to infection in patients with CGD is caused by the inability of their phagocytes to kill ingested microorganisms; during phagocytosis, CGD phagocytes lack the capacity to generate bactericidal oxygen products because of NADPH oxidase deficiency (3). This oxidase is a multicomponent complex that consists of membrane-bound cytochrome $b$ and several cytosolic components (4). Recent investigation has revealed that cytochrome $b$ is a heterodimer composed of a 91$\mathrm{kD}$ glycoprotein (heavy chain) and a $22-\mathrm{kD}$ polypeptide (light chain) (4), and the gene for the heavy chain is the site of mutations responsible for the X-linked form of CGD (5). Although the molecular genetic defects have been studied in various hereditary diseases $(6-10)$, this kind of examination of X-linked CGD has been done in only a few cases with a point mutation of the cytochrome $b$ heavy-chain gene $(11,12)$. Even in the analysis of genetic heterogeneity in X-linked CGD, only a few studies at the molecular level have been reported in a limited

Received August 6, 1991; accepted January 3, 1992.

Correspondence: Tadashi Ariga, M.D. The Department of Pediatrics, Hokkaido University School of Medicine, N-14, W-5, Kita-Ku, Sapporo 060, Japan.

Supported by a grant from the Ministry of Health and Welfare, Japan. number of families $(5,11-13)$. In this study, we found three novel mutations in three independent families with $\mathrm{X}$-linked CGD detected by Southern blot analysis.

\section{MATERIALS AND METHODS}

Patients. We studied 12 patients from 11 different families (two of whom had the same maternal grandmother) with Xlinked CGD. Patients were all male and ranged from 1 to $15 \mathrm{y}$ of age (Table 1). One patient died before the study was undertaken; his frozen leukocyte pellet was used for the analysis. Diagnosis of CGD was based on family and personal history of recurrent pyogenic infections, on the findings of defective reduction of nitroblue tetrazolium and impaired chemiluminescence production by neutrophils. Twelve CGD patients were considered to have X-linked inheritance, according to their family histories and familial granulocyte functions, studied by the tests mentioned above. All mothers of the patients were supposed to be carriers for the disease from the results of subnormal reduction of nitroblue tetrazolium. DNA samples from available family members and healthy controls were also used for the analysis.

Western blot analysis. Detection of cytochrome $b$ heavy- and light-chain protein of neutrophils was performed as described (14). Antibodies used for heavy and light chains were anti- $\mathrm{L}_{c}$ (against amino acid residues 150-172 of heavy-chain residues) and anti-S $\mathrm{S}_{C}$ (against amino acid residues 175-194 of light-chain residues), respectively (14).

Preparation of genomic DNA and Southern blot analysis. High molecular weight DNA was extracted from peripheral blood leukocytes, or from established Epstein-Barr virus-transformed B cell lines using standard methods described elsewhere (15). DNA was quantified by absorbance at $260 \mathrm{~nm}$, and $7.5 \mu \mathrm{g}$ of DNA was digested with restriction enzymes (BamHI, BglII, EcoRI, HindIII, PstI, PvuII, SacI, and TaqI) (Takara Shuzo Co., Ltd., Kyoto, Japan). DNA samples were separated by electrophoresis on $0.8 \%$ agarose gels and blotted onto nitrocellulose (Schleicher \& Schuell Inc., Keene, NH) after treatment according to Wahl et al. (16). Prehybridization and hybridization were carried out at $42^{\circ} \mathrm{C}$ in a mixture containing $50 \%$ formamide. The cytochrome $b$ heavy-chain cDNA, approximately $1.8 \mathrm{~kb}$ (encompassing the entire coding region) cloned into the HindIII site in the $\mathrm{psV}$ vector, was kindly provided by Dr. Stuart $\mathrm{H}$. Orkin (Harvard Medical School, Boston, MA). The full-length cDNA $(1.8 \mathrm{~kb})$ used as a probe was purified from the gel, after the vector including the cDNA had been digested with HindIII, and was separated by electrophoresis. A $5^{\prime}$ fragment $(0.2 \mathrm{~kb}$; HindIII/Pst I), and a $3^{\prime}$ fragment $(0.3 \mathrm{~kb}$; HindIII/EcoRI of the full-length cDNA were also purified from the gel and used as probes. Labeling of the probes was achieved by oligolabeling methods (17) (Takara Shuzo Co., Ltd.) using $\alpha-\left[{ }^{32}\right.$ P]dCTP (Amersham Corp., Arlington, IL). After washing the filter, hybridization patterns were visualized by autoradiography. 


\section{RESULTS}

Western blot analysis revealed that all the patients studied showed no cytochrome $b$ heavy- or light-chain protein, except for CGD patient 5. Patient 5 was revealed to have both cytochrome $b$ components (Table 1). The analysis for patient 10 could not be performed because he died before the study was undertaken.

The abnormal restriction fragment patterns of the cytochrome $b$ heavy-chain gene were detected in three patients with X-linked CGD (CGD1, CGD4, and CGD5). The other nine patients (eight different families) did not show any abnormalities detectable by Southern blot analysis.

DNA from CGD patient 1 showed absence of one band (1.8 $\mathrm{kb})$ after TaqI digestion and hybridization with cytochrome $b$ heavy-chain cDNA (Fig. 1, lane 1). DNA samples from more

Table 1. Summary of X-linked patients studied

\begin{tabular}{|c|c|c|c|c|c|}
\hline \multirow[b]{2}{*}{ Patient } & \multirow[b]{2}{*}{$\operatorname{Sex}$} & \multirow[b]{2}{*}{ Age } & \multicolumn{2}{|c|}{$\begin{array}{c}\text { Cytochrome } b \\
558\end{array}$} & \multirow{2}{*}{$\begin{array}{c}\text { Abnormal pattern of } \\
\text { cytochrome } b \text { heavy- } \\
\text { chain gene }\end{array}$} \\
\hline & & & $91 \mathrm{kD}$ & $22 \mathrm{kD}$ & \\
\hline CGDl & $\mathrm{M}$ & 15 & - & - & Yes $($ TaqI $)$ \\
\hline CGD2* & $\mathrm{M}$ & 12 & - & - & No \\
\hline CGD3* & $\mathrm{M}$ & 7 & - & - & No \\
\hline CGD4 & $\mathrm{M}$ & 1 & - & - & Yes (several enzymes) \\
\hline CGD5 & $M$ & 7 & + & + & Yes $(P s t \mathrm{I})$ \\
\hline CGD6 & $\mathrm{M}$ & 15 & - & - & No \\
\hline CGD7 & M & 12 & - & - & No \\
\hline CGD8 & $\mathbf{M}$ & 7 & - & - & No \\
\hline CGD9 & M & 6 & - & - & No \\
\hline CGD10 + & M & 12 & $? \ddagger$ & $?+$ & No \\
\hline CGD11 & M & 12 & - & - & No \\
\hline CGD12 & M & 7 & - & - & No \\
\hline
\end{tabular}

* Have the same maternal grandmother.

$\dagger$ Died before this study.

$¥$ Not studied.

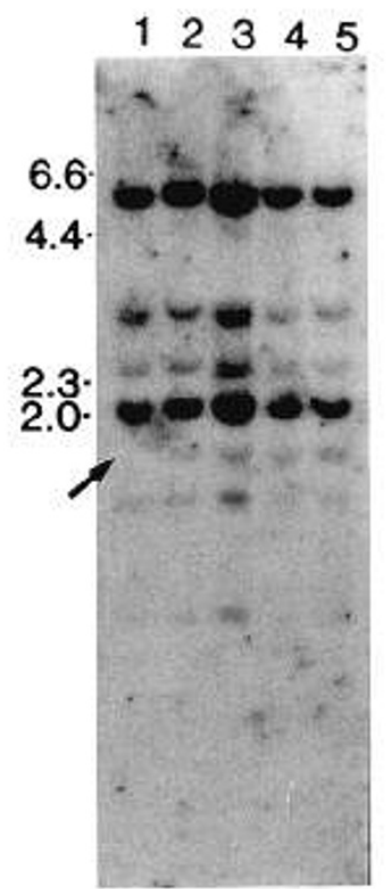

Fig. 1. Southern blot analysis of TaqI-digested DNA from CGD family 1. DNA samples from the patient (lane 1), his family members (his mother, lane 2; his sister, lane 3; his healthy brother, lane 4), and normal control (lane 5) were digested with TaqI and hybridized with the full-length cytochrome $b$ heavy-chain cDNA. The $1.8 \mathrm{~kb}$ band was missing in lane 1 (indicated by an arrow). than 20 healthy controls and the healthy brother of patient 1 (Fig. 1, lane 4) did not have the same abnormality with this enzyme. No other abnormal patterns were observed with any other restriction enzymes that we used in this patient.

The DNA of CGD patient 4 revealed the abnormality of the cytochrome $b$ heavy-chain gene with several restriction enzymes on Southern blot analysis (Fig. 2). For example, the patient's DNA revealed novel bands of 3.7 (HindIII; Fig. $2 A$, lane 1 ), 6.0 (PvulII; Fig. 2B, lane 1), and $11.5 \mathrm{~kb}$ (SacI; Fig. 2C, lane 1), none of which was seen in control individuals. Instead of new bands, 4.5- (HindIII; Fig. 2A, lane 2), 4.7- (PvuII, Fig. 2B; lane 2 ), and 10-kb (SacI; Fig. 2C, lane 2) bands, all of which were observed in controls, were missing. DNA from his mother (Fig. 3, lane 2) and his maternal grandmother (Fig. 3, lane 3) showed the same abnormal bands mentioned above, together with bands that were observed in controls but not detected in the patient (Fig. 3, lane 4). His sister's DNA did not show the abnormal bands on Southern blot analysis (Fig. 3). To begin to localize the mutation responsible for the abnormal bands on the Southern blot analysis, the $5^{\prime}$ fragment $(0.2 \mathrm{~kb})$ or the $3^{\prime}$ fragment $(0.3$ $\mathrm{kb}$ ) of the 1.8-kb cytochrome $b$ heavy-chain cDNA was used for hybridization. The abnormality was revealed again with the $3^{\prime}$ probe of the cytochrome $b$ heavy-chain cDNA, after digestion with HindIII, PvuII (Fig. 4C), and SacI. No abnormal band was detected with the $5^{\prime}$ probe (Fig. $4 B$ ).

DNA from CGD patient 5 revealed the abnormal pattern with PstI using the full-length cytochrome $b$ heavy-chain cDNA probe. Two bands, approximately 11 and $2.0 \mathrm{~kb}$, were missing, and a 13-kb band, which was not detected in DNA from healthy controls, was observed on Southern blot analysis (Fig. 5, lane 1). His mother's DNA was revealed to have the $11-$ and the $2.0-\mathrm{kb}$ bands, together with the 13-kb band, when it was digested with PstI and hybridized with the full-length probe (Fig. 5, lane 2). No abnormal band was detected with any of the other restriction endonucleases that we used.
A

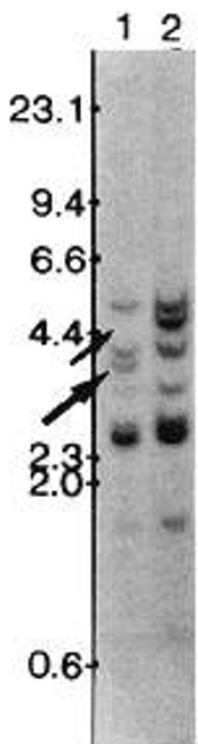

B

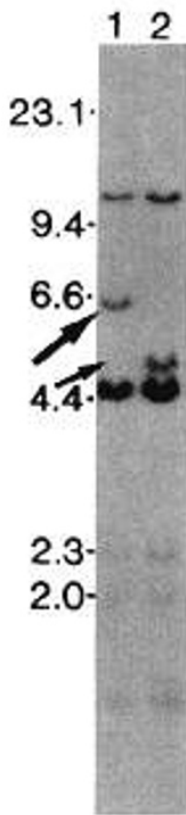

12

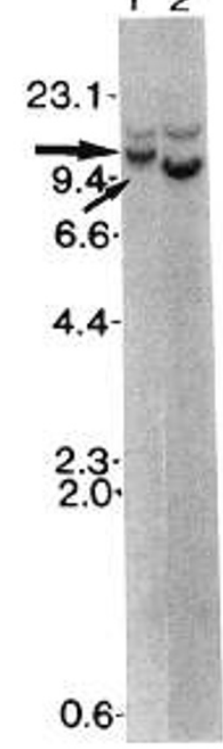

Fig. 2. DNA from CGD patient 4 (the patient, lane 1; normal control, lane 2) revealed the abnormal restriction fragment patterns of the cytochrome $b$ heavy-chain gene after digestion with several enzymes and hybridization with the full-length probe. Restriction enzymes used were HindIII $(A), P v u I I(B)$, and SacI $(C)$. Small arrows indicate missing bands, and large arrows indicated abnormal bands. 


\section{5}

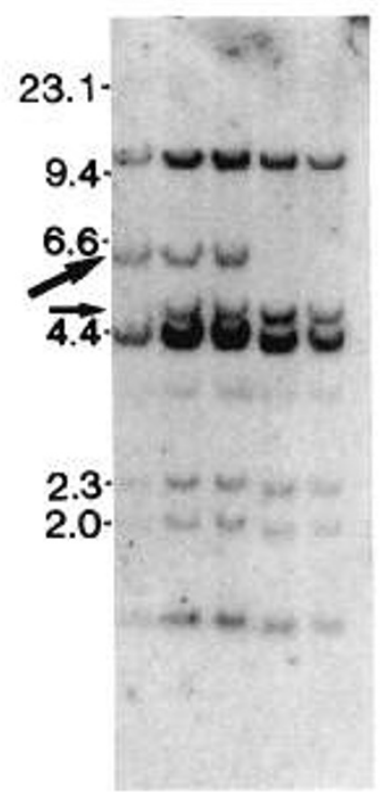

Fig. 3. Southern blot analysis of DNA samples from CGD family 4. $P v u$ II-digested DNA from the patient (lane 1), his mother (lane 2), his maternal grandmother (lane 3), his sister (lane 4), and normal control (lane 5) were hybridized with the full-length probe. A small arrow indicates a missing band, and a large arrow indicates an abnormal band.
A

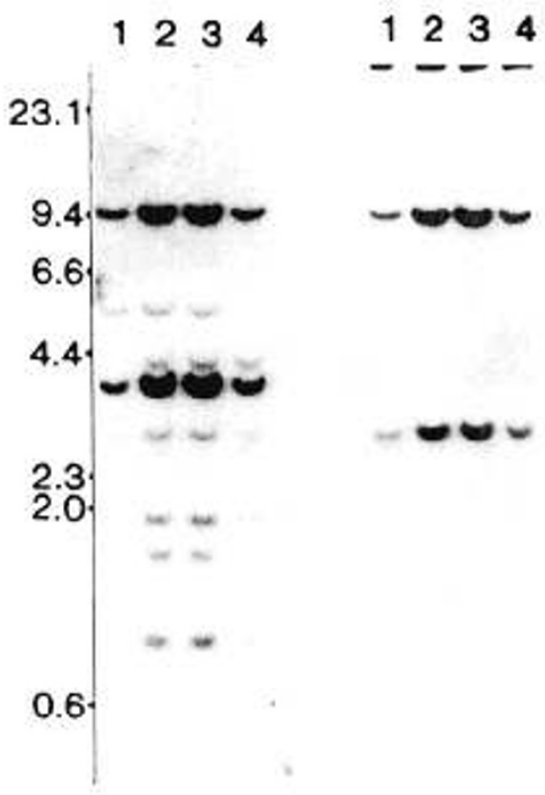

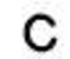

1234

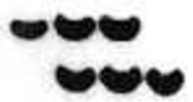

Fig. 4. Southern blot analysis of DNA samples from CGD patient 4 (lane 1), his mother (lane 2), his maternal grandmother (lane 3), and normal control (lane 4) using the full-length cytochrome $b$ heavy-chain cDNA and two different fragments of the cDNA. Each DNA sample was digested with $P v u \mathrm{II}$ and hybridized with the full cDNA $(A)$, with a $5^{\prime}$ fragment of the cDNA $(B)$, and with a $3^{\prime}$ fragment of the cDNA $(C)$. The $5^{\prime}$ and $3^{\prime}$ fragments of the cDNA were introduced by digestion of the full-length cDNA with $H$ indIII/Pst I and HindIII/EcoRI, respectively. The abnormal band was detected with the full cDNA and the 3 ' fragment of the cDNA.
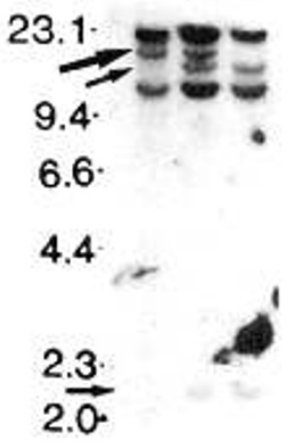

Fig. 5. Southern blot analysis of PstI-digested DNA samples from CGD patient 5 (lane 1), his mother (lane 2), and normal control (lane 3). Each DNA was digested with PstI and hybridized with the full-length cytochrome $b$ heavy-chain cDNA. The patient's DNA revealed the abnormal restriction fragment pattern. Two bands, approximately 11 and $2.0 \mathrm{~kb}$, were missing (indicated by small arrows). A novel 13-kb band, which was not detected in the normal control lane, was observed (indicated by a large arrow). His mother's DNA had all of the bands described above.

\section{DISCUSSION}

We have demonstrated the molecular genetic heterogeneity detectable on Southern blot analysis in three families with Xlinked CGD. The abnormal patterns of the cytochrome $b$ heavychain gene observed in the three CGD patients differed from each other. Southern blots of DNA from family members of the patients indicated that the abnormal patterns cosegregated with the disease. As reported recently (18), no restriction fragment length polymorphism was observed in healthy individuals with any of the restriction enzymes that we used in the study. It, therefore, seemed likely that the abnormal patterns on the Southern blot observed in patients were the results of mutations in the cytochrome $b$ heavy-chain gene responsible for the disease.

The DNA of CGD patient 4 showed the abnormal patterns of the cytochrome $b$ heavy-chain gene after digestion with several restriction enzymes and hybridized with the full-length cytochrome $b$ heavy-chain cDNA. His mother and maternal grandmother, who are presumed to be carriers of the disease, also had the same mutant gene (Fig. 3, lane 2 and 3). Furthermore, we were able to determine that his sister was not a carrier of the disease (Fig. 3, lane 4), because she did not have the same allele of the cytochrome $b$ heavy-chain gene that the patient possessed. Because the abnormal band was observed again using not the $5^{\prime}$ fragment, but rather the $3^{\prime}$ fragment of cytochrome $b$ cDNA on Southern blot analysis, the mutation responsible for the disease in the CGD patient 4 seemed to be located near, but not in, the portion corresponding to the $3^{\prime}$ fragment of cDNA. This mutation looked different from a partial deletion in the cytochrome $b$ heavy-chain gene, which has previously been reported $(5,13)$. Further molecular study will be necessary to characterize the mutation in CGD patient 4.

The mutation in CGD patient 1 seemed not to be a grossly detectable gene deletion or rearrangement of the cytochrome $b$ heavy-chain gene, because the abnormal band of the gene was observed only after digestion with TaqI. No abnormality was 
detected with any other endonuclease that we used. Because the TaqI recognition site "TCGA" includes dinucleotide "CpG," which is the hot spot for the point mutation (19), the mutation in CGD patient 1 might be located at one of four TaqI sites in the exon in the cytochrome $b$ heavy-chain gene (5). The absence of the $1.8-\mathrm{kb}$ band in this patient was more likely due to a change in size and overlapping of one of the bands observed on the film. The possibility of a partial deletion in the gene was small, because we could not detect the abnormality of the gene with any other restriction enzymes. DNA samples from more than 20 normal controls and the healthy brother of CGD patient 1 (Fig. 1, lane 4) did not have the same pattern as the patient with this enzyme. This was consistent with the hypothesis that this pattern cosegregated with the disease and was the result of the mutation responsible for the disease. However, we cannot exclude the possibility that this abnormality on the Southern blot was linked to the disease but was not due to the mutation responsible for the disease.

Although CGD patient 5 was shown to have both cytochrome $b$ chains on Western blot studies (we also revealed that both of the cytosolic factors ( 47 and $65 \mathrm{kD}$ ) of the patient's granulocytes were positive on Wesiern blot analysis (not shown)], we think that the mutation responsible for the disease in the patient is in the cytochrome $b$ heavy-chain gene because we detected the abnormal restriction length pattern of the gene, which was not seen in normal individuals. The mutation of the patient that seemed to result in dysfunctional cytochrome $b$ protein also seemed not to have a gross abnormality in the cytochrome $b$ heavy-chain gene structure. The mutation of the gene was detected only after digestion with Pst I, and control DNA samples did not show any polymorphic pattern of the gene with this enzyme. Furthermore, Southern blot study of the DNA of the patient's mother indicated that this Pst I abnormality cosegregated with the disease. Because she was a carrier of the disease, she had the same mutation in the cytochrome $b$ heavy-chain gene together with the normal gene. This Pst I abnormality would be very helpful to determine carrier state of the disease in this family. Further study to characterize the mutation in this patient would be very helpful to understand the functional domain of cytochrome $b$ heavy chain, and it is presently underway.

The other nine patients from eight families did not reveal any abnormal pattern of the cytochrome $b$ heavy-chain gene on Southern blot analysis with any of the enzymes that we used. This suggests that these mutations also involve a small portion of the gene. Thus, 10 of 11 families with X-linked CGD in this study appeared not to have a grossly detectable genetic mutation. This rate is consistent with a previous report that the abnormality of the cytochrome $b$ heavy-chain gene on Southern blot study, which resulted from mutations, was observed in only one of 18 different families with X-linked CGD (5). Further analysis should be done for the other eight families to define whether they have the same mutation or their mutations are heterogeneous.
Acknowledgment. The authors thank Drs. M. Hirota, Y. Wagatsuma, Y. Ogasawara, T. Yamanaka, and T. Togashi for allowing us to study their patients. We also thank Drs. S. H. Orkin and M. C. Dinauer for providing us with the cytochrome $b$ heavy-chain cDNA.

\section{REFERENCES}

1. Tauber AI, Borregaard N. Simons E. Wright J 1983 Chronic granulomatous disease: a syndrome of phagocyte oxidase deficiencies. Medicine 62: 286-309

2. Orkin SH 1989 Molecular genetics of chronic granulomatous disease. Annu Rev Immunol 7:277-307

3. Shurin SB, Cohen HJ, Whitin JC. Newburger PE 1983 Impaired granulocyte superoxide production and prolongation of the respiratory burst due to a low affinity NADPH-dependent oxidase. Blood 62:564-571

4. Smith RB, Curnutte JT 1991 Molecular basis of chronic granulomatous disease. Blood 77:673-686

5. Royer-Pokora B, Kunkel LM, Monaco AP. Goff SC. Newburger PE, Baehner RL, Cole FS, Curnutte JT, Orkin SH 1986 Cloning the gene for an inherited human disorder-chronic granulomatous disease-on the basis of its chromosomal location. Nature 322:32-38

6. Nienhuis AW. Anagnou NP. Ley TJ 1984 Advances in thalassemia research. Blood 63:738-758

7. Wilson JM, Stout JT, Pallela TD, Davidson BL, Kelley WN, Caskey CT 1986 A molecular survey of hypoxanthineguanine phosphoribosyltrasferase deficiency in man. J Clin Invest 77:188-195

8. Prochownik EV. Autonarakis VS, Bauer KA. Rosenburg RD, Fearon ER. Orkin SH 1983 Molecular heterogeneity of inherited antithrombin III deficiency. N Engl J Med 308:1449-1452

9. Gitschier T, Wood WI, Tuddenham MA. Shuman TM, Goralka TM, Chen EY, Lawn RM 1985 Detection and sequence of mutations in the factor VIII gene of haemophiliacs. Nature 315:427-430

10. Ariga T. Carter P. Davis III AE 1990 Recombinations between Alu repeat sequences that result in partial deletions within the $\mathrm{Cl}$ inhibitor gene. Genomics 8:607-613

11. Dinauer MC. Curnutte JT, Rosen H, Orkin SH 1989 A missense mutation in the neutrophil cytochrome $b$ heavy chain in cytochrome-positive X-linked chronic granulomatous disease. J Clin Invest 84:2012-2016

12. Bolscher BGJM. Boer M. Klein A, Weening RS, Roos D 1991 Point mutations in the $\beta$-subunit of cytochrome $b 558$ leading to X-linked chronic granulomatous disease. Blood 77:2482-2487

13. Alison P. O'Reilly MAJ. Malcolm S. Levinsky RJ, Kinnon C 1990 RFLP and deletion analysis for $\mathrm{X}$-linked chronic granulomatous disease using the cDNA probes: potential for improved prenatal diagnoses and carrier determination. Blood 76:820-824

14. Imajoh-Ohmi S. Tokita K. Ochiai H. Nakamura M. Kanegasaki S 1992 Topology of cytochrome $b_{s s k}$ in neutrophil membrane analyzed by antipeptides and proteolysis. J Biol Chem (in press)

15. Whitehead AS, Woods DE. Fleishnic JE. Chin JE. Yunis EJ. Katz AJ. Gerald PS, Alper CA. Colten HR 1984 DNA polymorphism of the C4 genes: a new marker for analysis of the major histocompatibility complex. N Engl J Med 310:88-91

16. Whal GM, Stern M, Stark GR 1979 Efficient transfer of large DNA fragments from agarose gels to diazobenzoloxymethal paper and rapid hybridization using dextran sulfate. Proc Natl Acad Sci USA 76:3683-3687

17. Feinberg AP, Volgelstein B 1983 A technique for radiolabeling DNA restriction endonuclease fragments to high specific activity. Anal Biochem 132:6-13

18. Battat L. Francke U 1989 Nsi I RFLP at the X-linked chronic granulomatous disease locus (CYBB). Nucleic Acids Res 17:3619

19. Cooper DN, Youssoufian H 1988 The CpG dinucleotide and human genetic disease. Human Genet 78:151-155 